\title{
Subclinical gut inflammation in ankylosing spondylitis
}

\author{
Francesco Ciccia $^{\mathrm{a}}$, Aroldo Rizzo ${ }^{\mathrm{b}}$, and Giovanni Triolo ${ }^{\mathrm{a}}$
}

\begin{abstract}
Purpose of review
Subclinical gut inflammation has been described in a significant proportion of patients with ankylosing spondylitis (AS), up to $10 \%$ of them developing it during the time of clinically overt inflammatory bowel disease. Histologic, immunologic, and intestinal microbiota alterations characterize the AS gut.
\end{abstract}

\section{Recent findings}

Microbial dysbiosis as well as alterations of innate immune responses have been demonstrated in the gut of AS. Furthermore, a growing body of evidence suggests that the gut of AS patients may be actively involved in the pathogenesis of AS through the production of proinflammatory cytokines, such as IL-23p19, and the differentiation of potentially pathogenic innate lymphoid cells producing IL-22 and IL-17. Finally, a strong correlation between the presence of subclinical gut inflammation and the degree of spine inflammation have been also proved in AS.

\section{Summary}

Subclinical gut inflammation and innate immune responses in AS may be considered a possible consequence of microbial dysbiosis. Relationships between cause and effect remain, however, to be answered.

\section{Keywords}

ankylosing spondylitis, gut inflammation, interleukin-17, interleukin-23, innate lymphoid cells

\section{INTRODUCTION}

Subclinical gut inflammation has been described in up to $60 \%$ of patients with spondyloarthritis (SpA) [1], with up to $10 \%$ of those with more pronounced gut inflammation evolving in a clinically overt Crohn's disease [2]. On the basis of these epidemiologic findings, SpA-associated gut inflammation has been initially thought as a preclinical model of Crohn's disease where to study early immunological alterations related to Crohn's disease. This point of view, however, seems to be too simplistic since a growing body of recent evidence suggest that gut inflammation, at least in ankylosing spondylitis (AS) patients, is not an epiphenomenon of the ongoing systemic inflammatory process but rather an important etiological event that may actively participate in the pathogenesis of AS. This article will focus on recent studies regarding the innate and adaptive immune responses occurring in the inflamed gut of AS patients.

\section{TEXT OF REVIEW}

\section{Histology}

Two main types of gut inflammation have been described in AS patients: acute inflammation, resembling a self-limiting bacterial enterocolitis, and chronic inflammation, displaying altered intestinal architecture with strong infiltration of mononuclear cells eventually aggregated in lymphoid follicles, resembling the ileocolitis seen in Crohn's disease [1]. Behind the architectural alterations described in AS gut, other histologic findings seem to characterize the inflamed ileum of AS. Relevant epithelial alterations are in fact present in AS gut, mainly characterized by goblet cells hyperplasia (with increased mucins production) [3] and activation of paneth cells, producing high levels of antimicrobial peptides [4] and proinflammatory cytokines such as IL-23 [5]. Other histologic alterations include the presence of detachment of epithelial cells from the basal membrane and the

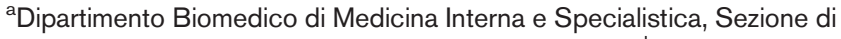

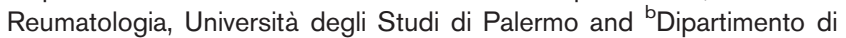
Oncoematologia, Azienda Ospedaliera Ospedali riuniti Villa SofiaCervello, Palermo, Italy

Correspondence to Professor Giovanni Triolo, Department of Internal Medicine, Division of Rheumatology, Piazza delle Cliniche 2, 90127 Palermo, Italy. Tel: +390916552137; e-mail: giovanni.triolo@unipa.it
}

Curr Opin Rheumatol 2016, 28:89-96

DOI:10.1097/BOR.0000000000000239 


\section{KEY POINTS}

- Intestinal dysbiosis occurs in AS.

- The inflamed gut of AS is the main site of production of IL-23.

- Activation and expansion of ILCs occur in the gut of AS.

- Gut-derived ILCs accumulate in the site of inflammation in AS.

occurrence of vasculitic lesion, mainly represented by intense hemorrhagic extravasation in the context of lamina propria (Fig. 1) [6]. Although we cannot exclude that genetic factors may be responsible for these alterations, similar epithelial damages have been described as a consequence of the response of epithelial cells to bacterial toxins $[7,8]$.

\section{Intestinal dysbiosis in ankylosing spondylitis}

Crohn's disease is one of the major inflammatory bowel diseases. Genetic studies have demonstrated that several genes, most of which were involved in immune responses, appear to predispose for Crohn's disease [9]. However, genes alone cannot explain the pathogenesis of Crohn's disease. Increasing evidence suggests that the intestinal microbiota may play a role in initiating and maintaining the phenotype of Crohn's disease. A number of studies have

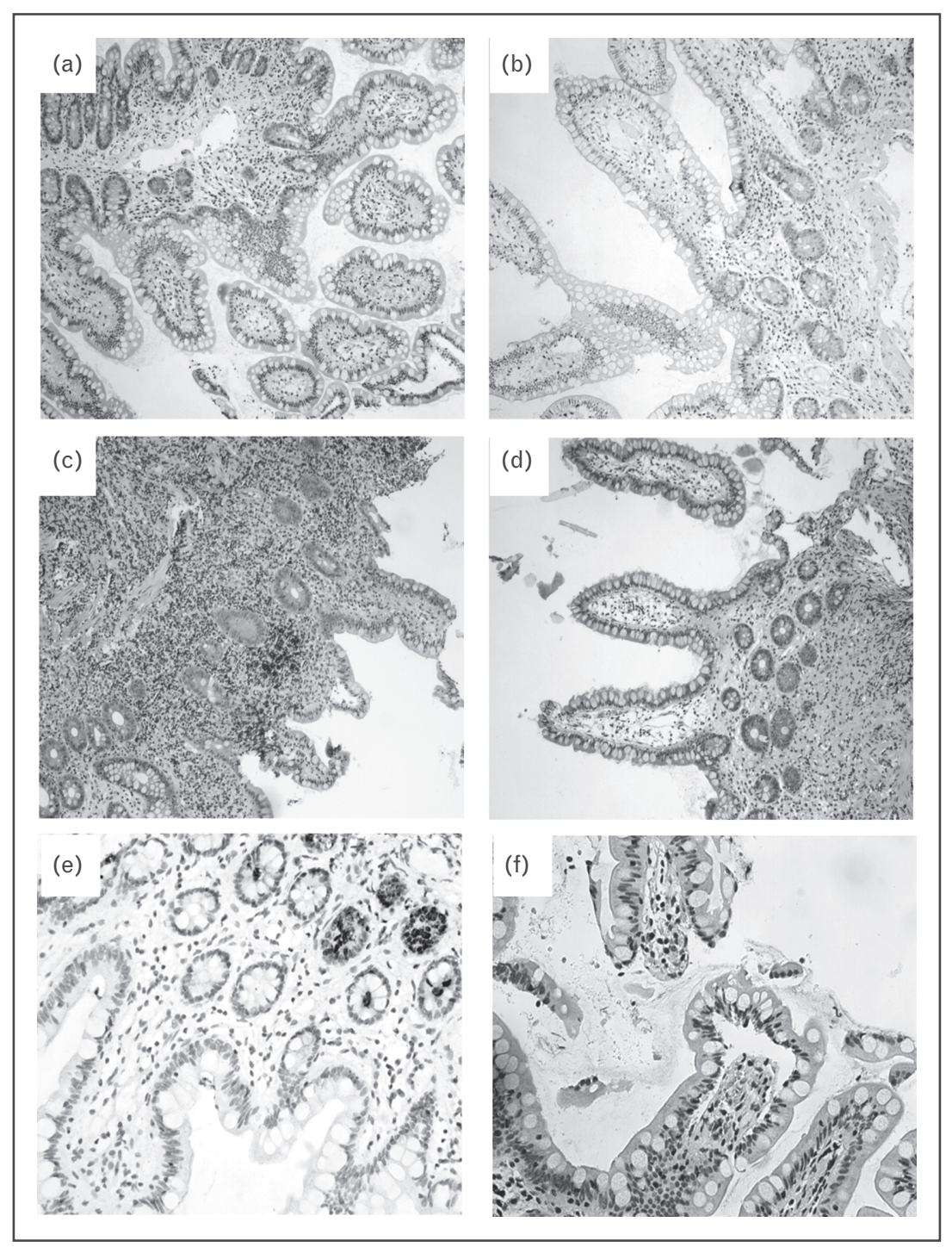

FIGURE 1. Subclinical gut inflammation in AS patients. (a and b): Acute intestinal inflammation in AS. (c and d) Chronic intestinal inflammation in AS patients with histologic findings of Crohn's disease (c). (e) Increased expression of a-defensin 5 in AS gut, as a marker of paneth cells activation. (f) Detachment of epithelium from basal membrane and hemorrhages in AS gut. (a-d) Original magnification 100×. (e and f) Original magnification 400×. AS, ankylosing spondylitis. 
in fact demonstrated the presence of significant alteration in Crohn's disease gut microbiome mainly characterized by the occurrence of opportunistic pathogens, the presence of dysbiosis, the presence of functional alterations in the commensal bacteria, and the host inability in containing commensal microorganisms probably because of genetic factors (reviewed in [10]). The resulting continuous antigenic stimulation may activate pathogenic $\mathrm{T}$ effector cells that may be in turn responsible of the chronic intestinal inflammation.

Human leukocyte antigen B27 (HLA-B27) is a major risk factor for AS [11]. The role of HLA-B27 in shaping the gut microbiome has been studied by the Rosenbaum's group demonstrating, by using biome representational in-situ karyotyping and $16 \mathrm{~S}$ ribosomal ribonucleic acid gene sequencing, that Lewis rats transgenic for HLA-B27 and human $\beta 2$-microglobulin, had significant differences in the cecal microbiota compared with wild-type Lewis rats [12]. The role of gut microbiome in AS pathogenesis is also suggested by many studies [13-15] that demonstrate, in patients and first-degree relatives, an increased intestinal permeability. In this regard, it is probable that AS may be triggered by common environmental (microbial) agents in genetically susceptible individuals. Costello et al. [16"'] recently performed culture-independent microbial community profiling of terminal ileum biopsy specimens from AS patients and healthy controls to characterize and investigate any differences in the gut microbiome. Terminal ileal biopsies obtained from AS patients were analyzed for their microbial profiles. Ileal microbial communities of patients with AS differed significantly from healthy controls, being characterized by higher abundance of five bacteria families: Lachnospiraceae, Veillonellaceae, Prevotellaceae, Porphyromonadaceae, and Bacteroidaceae.

The evidence of altered microbiome in other SpA phenotypes reinforces the role of intestinal dysbiosis in this group of related disorders. Altered microbiota, characterized by reduced Faecalibacterium prausnitzii and Lachnospiraceae families and an increase in Bifidobacterium, has been recently demonstrated in enthesitis-related arthritis associated with an abnormal humoral immune response [17]. Furthermore, patients with psoriatic arthritis had a lower relative abundance of multiple intestinal bacteria displaying a microbiota profile similar to that previously described in patients with inflammatory bowel disease and associated with changes in specific inflammatory proteins [18]. Although the findings coming from these works, are interesting studies are needed to specifically address the effect of intestinal dysbiosis on innate immune responses and tissue inflammation and to value if the changes in intestinal microbial composition are because of host genetics.

\section{Macrophage signature in ankylosing spondylitis gut}

As the dysbiosis observed in AS, it is likely that host factors, such as cells of the innate immune system, may predispose for the establishment of an altered intestinal microbiome. Intestinal macrophages are important components of intestinal immunity and essential for the maintenance of mucosal homeostasis [19]. Intestinal macrophages are unresponsive to bacterial stimuli, displaying a reduced expression of Toll-like receptors (TLRs) and other functional receptors necessary for macrophage activation (i.e. CD14) [20]. In Crohn's disease, this anergic phenotype is lost and CD14-expressing macrophages accumulate in the inflamed lamina propria where they release proinflammatory mediators and are involved in the induction of Th1 and Th17 responses [21,22].

Macrophages are not a homogeneous population and, according to their phenotype and function, different pathways of macrophage activation have been described in humans. Following the Th1/ Th2 nomenclature, macrophages are subdivided in prevalent M1 (classical/proinflammatory) or M2 (alternative/antiinflammatory) types, representing, however, the extremes of a continuum of activation states [23]. Another subset of macrophages, possessing aspects of both definitions consistent with an immune regulatory phenotype, the so-called resolution phase macrophages (rMs) has been recently described [24]. Impairment of M1-derived mediators and expansion of M2 macrophages have been demonstrated in the colon and synovial fluid of AS patients $[25,26]$. Interestingly, in the AS facet joints IL-23 seems to be predominantly produced by M2 but not M1 macrophages [27]. In the ileum of AS patients, balanced macrophage activation seems to be present with $\mathrm{CD} 14^{+}$macrophages being virtually absent and classically activated M1 macrophages (iNOS ${ }^{+} \mathrm{IL}_{-10}{ }^{-} \mathrm{CXCL}-9^{+}$) expanded. Furthermore, a significant increase of $\mathrm{iNOS}^{+} \mathrm{IL}_{-}-10^{+}$cells (defined as rMs) and $\mathrm{CD}_{163^{+}}$ileal macrophages (M2 macrophages), strictly correlated with the expression of IL33 , a Th2 cytokine involved in M2 polarization, is also present in AS gut [28]. Expansion of rMs and of M2 macrophages, incompetent to eradicate intestinal bacteria, may be responsible for the persistence of intestinal dysbiosis and for lack of excessive local inflammation observed in the ileum of AS patients. The aberrant production of IL-23 by M2 macrophages, described in the spine of AS patients, and 
their specific role in modulating innate and adaptive immune responses need to be better investigated.

\section{Immunologic signature of ankylosing spondylitis gut}

According to M1 deficient polarization, an impaired Th1 profile with a possible Th2 polarization has been reported in gut lamina propria lymphocytes from patients with AS [5,29]. Immunologically, however, the gut of AS seems to be more complexly signed. IL-23 in particular has recently emerged as a key cytokine involved in the regulation of innate and adaptive immune responses in AS [30]. IL-23 mainly acts as a regulator of the maturation of autoreactive $\mathrm{T}$ cells producing IL-17 (Th17), and promotes chronic inflammation dominated by IL-17, IL-6, IL-8, and TNF- $\alpha$, as well as by neutrophils and macrophages [31].

Although in Crohn's disease patients, the IL-17/ IL-23 axis has been proved to be particularly activated, targeting Th17 responses via secukinumab (anti-IL-17A) resulted in worse outcomes [32]. The apparently paradoxical effect of IL-17 blocking agents may suggest critical differences between IL-17 itself (mainly produced by cells of the innate immune system), possibly involved in tissue protective responses, and the highly pathogenic Th17 cells. However, we cannot exclude that activation of different immune pathways following IL-17 inhibition, such as Th1 or Th2 2 cells, and/or modifications in the intestinal microbiome induced by loss of control by IL-17, may also be responsible for the failure of anti-IL-17 therapy.

Behind the role of IL-23 in the modulation of the Th17 pathway, recent reports identify an important role for IL-23 in directly modulating the innate intestinal immune system by activating and expanding the so-called innate lymphoid cells (ILCs) of type $3\left[33^{-*}, 34\right]$. IL-23 upregulation is present in the terminal ileum of AS patients, mainly in the context of paneth cells, and is not accompanied by a clear Th17 polarization [5] indicating a role of this proinflammatory cytokine in directly driving intestinal immune responses.

Although the regulation of IL-23 in AS gut is not completely understood, a pathway of innate immune system (the so-called macroautophagy or autophagy) may be implicated in IL-23 modulation [35]. Autophagy is a basic cellular machinery responsible in eukaryotic cells for bulk degradation of cellular constituents to provide energy resources to the cells, also acting by directly eliminating intracellular microbes or their products [36]. Another function of autophagy is connected to its ability to target improperly folded proteins for degradation in close connection with the endoplasmic reticulum (ER) stress response known as the unfolded protein response (UPR) [37]. Autophagy seems to be differentially regulated in the gut of AS patients where a strong and significant upregulation of autophagy-related 16-like 1 (ATG16L1), immunity-related GTPase family $M$ protein (IRGM), and microtubule-associated proteins $1 \mathrm{~A} / 1 \mathrm{~B}$ light chain 3A (MAP1LC3A) together with a decreased expression of genes involved in the so-called chaperonemediated autophagy occur. In particular, expression levels of ATG16L1, IRGM, and MAP1LC3A seem to be correlated with IL-23p19 in AS patients with chronic gut inflammation and inhibition of autophagy and chaperone-mediated autophagy appears sufficient, in the presence of lipopolysaccharide, to reduce the percentage of IL-23 expressing cells at the same time increasing the mRNA levels for IL23p19 in the lamina propria mononuclear cells (LPMCs) [38"']. Although activation of autophagy has not been demonstrated in the peripheral blood and synovial tissues of AS [39], our findings seem to indicate this pathway as one of the potential immunological factors regulating the production and secretion of IL-23 in AS in the gut.

Although these findings are of interest, linking bacterial innate immune responses to IL-23 production, other pathways might be implicated in the production of IL-23. In this regard, UPR as a consequence of HLA-B27 misfolding has been proposed, in murine models of AS, as a main pathway involved in IL-23 production [40]. This model, however, has not been confirmed in humans where UPR seems to be not significantly modulated [41]. Interestingly, free heavy chain misfolding occurs in the gut of AS patients without any increased expression levels of UPR genes, indicating that probably UPR in human gut is strictly modulated by other immunological pathways [38"']. In this regard, our data indicate that inhibition of autophagy and chaperone-mediated autophagy increases in AS LPMCs the levels of misfolded heavy chains and a clear upregulation of UPR genes [42]. As our results support the presence of HLA-B27 misfolding, we cannot obviously exclude that ER stress caused by HLAB27 in the gut of AS patients may contribute to the intense activation of autophagy, which in turn may further limit the full UPR activation by enhancing the removal of misfolded protein.

\section{Innate immune responses in ankylosing spondylitis gut}

The role of IL-23p19 in driving AS pathogenesis seems to be linked to its ability in modulating IL-17 and IL-22 production, two cytokines actually 
considered important actors in driving spinal inflammation and osteoproliferation [43]. IL-23responsive entheseal resident $\mathrm{T}$ cells producing IL-17 and IL-22 have been observed in a murine model of AS and correlated to AS pathogenesis [43]. The exact nature of these cells and their developmental origin remain elusive, also sharing immunological similarities with specific subsets of ILCs.

ILC populations are specialized effector cells involved in the regulation of innate immunity and inflammation through the secretion of specific cytokines and chemokines. ILCs are characterized by the absence of recombined antigen-specific receptors, the absence of specific markers associated with other immune cell lineages and a lymphoid morphology [44]. Based on their cytokine repertoire, ILCs are classified into three groups (ILC1, ILC2, and ILC3): ILC1s express the transcription factor T-bet, produce IFN- $\gamma$, and mediate immunity against intracellular pathogens and tumors; ILC2s produce IL-5 and IL-13; ILC3s are an important source of IL-22 and IL-17, in response to IL-23, express the retinoic acid, ROR- $\gamma$, and are required for mediating immunity to extracellular bacterial infections, also providing help to marginal zone B cells, and may also play a proinflammatory role $[45,46]$. ILC3s seem to be essentially mucosalrestricted cells, developmentally related to lymphoid tissue-induced cells (LTi) and dependent on IL-7 for their differentiation [40]. These ROR- $\gamma^{+}$cells are essentially involved in protective response by negatively regulating Th17 cells through the modulation of intestinal microflora [47,48"']. ILC3 may be further subdivided according to whether they express natural cytotoxicity receptors, such as the NKp44 receptor. Although the ligand for NKp44 in normal tissues has not yet been identified, it seems to bind to an unusual isoform of the mixed lineage leukemia 5 protein [49] and the continued engagement of NKp44 and cytokine receptors may induce a potent proinflammatory program in NKp $44^{+}$ILC3 cells [50]. Specifically in the gut of patients with AS, ILC3 expressing the natural cytotoxicity receptor NKp44 are significantly expanded and possibly involved in tissue protective mechanisms [3].

ILC populations were recently studied in the gut of AS patients [51"']. No significant expansion of ILC1 and/or ILC2 was observed. Conversely, a consistent expansion of NKp44 ${ }^{+}$ILC3 (Lyn ${ }^{-}$IL$23 \mathrm{R}^{+} \mathrm{NKp} 44^{+} \mathrm{Tbet}^{+} \mathrm{RORc}^{-}$) was demonstrated in patients with AS with acute and chronic gut inflammation significantly correlated with the disease activity as assessed by the Bath Ankylosing Spondylitis Disease Activity Index. Type 3 ILCs were also expanded in the gut, in the peripheral blood, in the synovial fluid and in the bone marrow of patients with AS and express the homing integrin $\alpha 4 \beta 7$. In addition, mucosal vascular addressin cell adhesion molecule 1 , the $\alpha 4 \beta 7$ ligand, was found to be highly represented in the high endothelial venules of the gut and in the inflamed bone marrow of AS, suggesting a role for this receptor in chemoattracting ILC3 at the site of active AS inflammation and a recirculation of ILC3 between the gut and the bone marrow (Fig. 2). In this regard, a better definition of the role of M2 macrophages in regulating ILC3 (for example, through the production of IL-23) needs to be defined.

ILC3s are developmentally related to LTi cells [47]. Aggregates of LTi in the small intestine of patients with AS were observed in close proximity to intestinal crypts [51"']. These structures, highly suggestive for cryptopatches, have been hypothesized to be sites of active postnatal extrathymic T cell development (as a consequence of intestinal bacteria colonization) and demonstrated only in the colon in humans $[52,53]$. Aggregates of c-kit ${ }^{+}$Thy$1^{+} \mathrm{CD}^{-}$cells were, however, demonstrated in the ileum of AS patients, in close proximity to intestinal crypts. In the gut of AS, paneth cells express IL-7 and coculture of LTi with epithelial cells isolated from patients with AS strongly induces the differentiation of ILC3, also increasing the expression of IL-17 and IL-22 [51""]. These results suggest a pivotal role of paneth cells in activating and amplifying intestinal innate immune responses in the gut of patients with AS resulting in active ILC3 differentiation (Fig. 2).

\section{Clinical associations between axial and subclinical gut inflammation in ankylosing spondylitis}

Bone marrow edema (BME) of the sacroiliac joints (SIJs) is actually considered to be a hallmark of axial SpA. A recently published study [54"'] specifically assessed the link between BME of the SIJs and gut inflammation and the correlation between BME and established disease activity parameters. The study was performed on 68 patients with axial SpA from the Gent Inflammatory Arthritis and spoNdylitis cohorT underwent ileocolonoscopy and MRI of the SIJs. Histopathological analysis and SPondyloArthritis Research Consortium of Canada (SPARCC) scores were performed. In this study, a significantly higher SPARCC score was observed in axial SpA patients showing chronic gut inflammation compared with axial SpA patients showing normal gut histology. By using a multiple linear regression model, the authors identified chronic gut inflammation to be independently associated with the extent of BME indicating a link between mucosal inflammation and axial disease in SpA. 


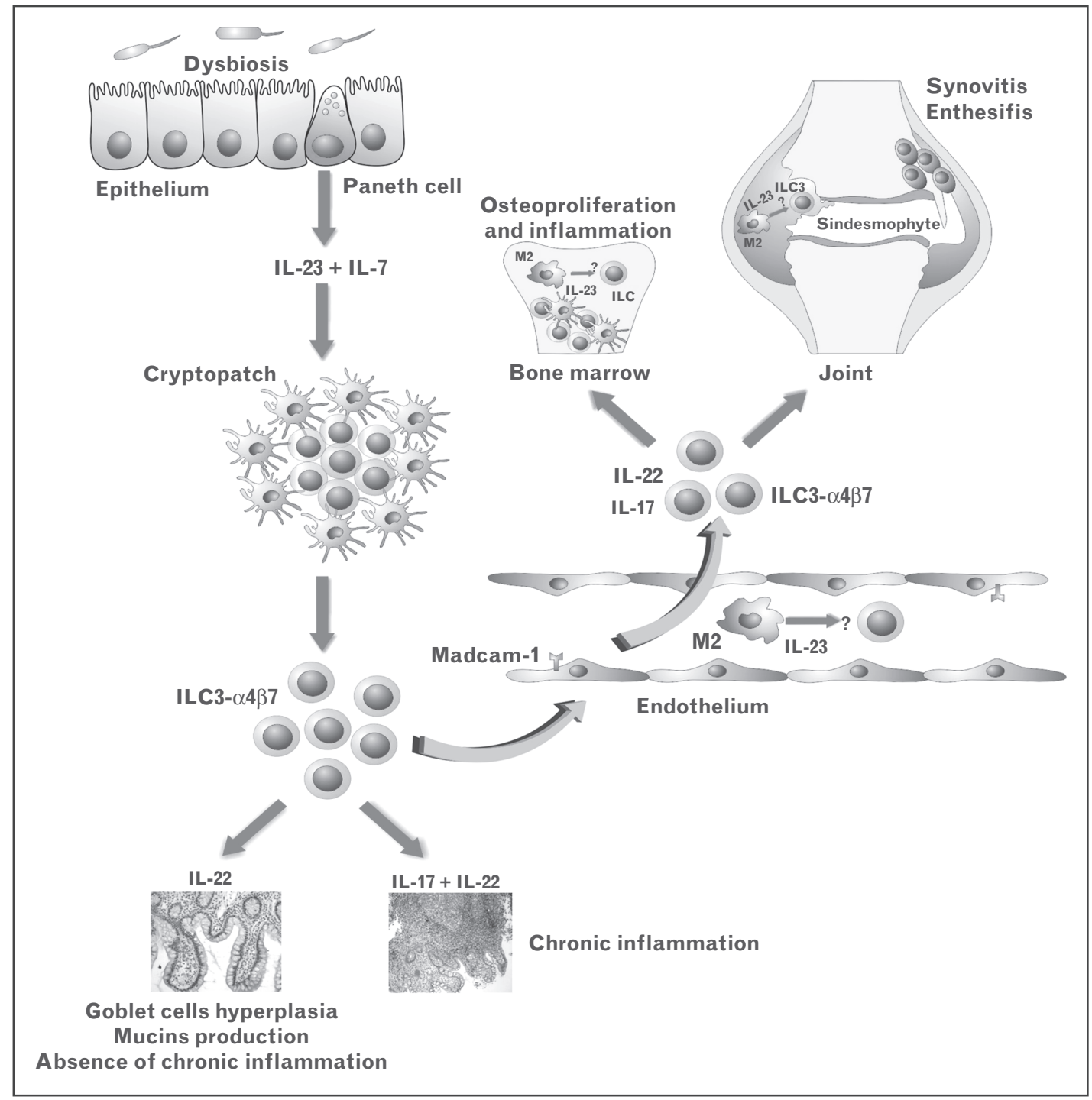

FIGURE 2. Role of microbiome and innate immune system on pathogenesis of ankylosing spondylitis. Altered gut microbiome may induce activation of paneth cells with the consequent release of proinflammatory cytokines such IL-23 and IL-7 that may induce the organization of lymphoid tissue inducer cells in aggregates, called cryptopatches. In this context may occur the differentiation of ILC3 producing IL-17 and IL-22. IL-22 alone may induce tissue protective responses on epithelial cells such as goblet cells hyperplasia and mucins production. Together with IL-17, IL-22 may be also responsible for the induction of tissue inflammation. ILC3 expressing $\alpha 4 \beta 7$ may migrate in the systemic circulation and, through the interaction with the specific counter-receptor MADCAM1, accumulate in the site of active inflammation such as peripheral joints and peripheral tissues rich in bone marrow such as entheses. By producing IL-22 and IL-17, ILC3 may be responsible for the induction of tissue inflammation. Outside of the gut activation of ILC could be supported by M2 macrophages. ILC3, innate lymphoid cells type 3; MAdCAM1, mucosal vascular addressin cell adhesion molecule 1.

\section{Murine models of gut inflammation in ankylosing spondylitis}

To study how host genetics may influence gut microbiota and the eventual relationship between microbiota and organ inflammation in SpA, Rehaume and coworkers [55] studied BALB/c ZAP70(W163C)-mutant (SKG) mice, TLR-4-deficient SKG mice, and wild-type BALB/c mice maintained under germ-free conditions, and recolonized with altered Schaedler flora. The mice were injected intraperitoneally with microbial $\beta$-1,3-glucan (curdlan) and arthritis, spondylitis, and ileitis assessed histologically. Microbiota content and response to curdlan varied according to whether $\mathrm{T}$ cell receptor signal strength was normal or was impaired because of the ZAP-70(W163C) mutation. Curdlan triggered acute inflammation regardless of the presence of the SKG allele or microbiota. However, no or limited microbiota content attenuated the severity of arthritis. In contrast, ileal IL-23 expression, ER stress, 
lymph node IL-17A production, goblet cell loss, and ileitis development were microbiota dependent. Ileitis but not arthritis was suppressed by microbiota transfer upon cohousing SKG mice with wild-type $\mathrm{BALB} / \mathrm{c}$ mice, as well as by TLR- 4 deficiency. The effects of IL-23 and IL-22 inhibition in IL-17A(-/-) in curdlan-treated SKG or BALB/c mice were assessed in another study [56]. In curdlan-treated SKG mice, arthritis, enthesitis, and ileitis were IL-23 dependent and enthesitis was specifically dependent on IL-17A and IL-22. IL-23 was induced in the ileum, where it amplified ER stress, goblet cell dysfunction, and proinflammatory cytokine production. IL-17A was pathogenic, whereas IL-22 was protective against ileitis. IL-22 ${ }^{+} \mathrm{CD}^{-}$innate-like cells were increased in LPMCs of ileitis-resistant BALB/c mice, which developed ileitis after curdlan injection and antiIL-22.

\section{CONCLUSION}

Subclinical gut inflammation may be the hidden place where, because of the continuous stimulation of the immune system by an altered microbiome, a chronic activation of the innate immune system occurs. In particular, ILCs activated in the gut may accumulate in the inflammatory lesions of patients with AS, potentially being a relevant early source of AS-associated effector cytokines and important drivers of mucosal and systemic inflammation. Insights into how modulation of ILCs for therapeutic purposes might affect the clinical outcome in AS patients are strongly awaited.

\section{Acknowledgements}

We would like to thank Dr Angelo Ferrante for his assistance with the study and in particular for helping in preparing Figure 2.

\section{Financial support and sponsorship}

This work was in part supported by a grant of the Ministero dell'Istruzione, Università e Ricerca Scientifica (MIUR), Italy.

\section{Conflicts of interest}

There are no conflicts of interest.

\section{REFERENCES AND RECOMMENDED \\ READING}

Papers of particular interest, published within the annual period of review, have been highlighted as:

- of special interest

m. of outstanding interest

1. De Vos $M$, Cuvelier $\mathrm{C}$, Mielants $\mathrm{H}$, et al. lleocolonoscopy in seronegative spondylarthropathy. Gastroenterology 1989; 96:339-344.
2. De Vos $M$, Mielants $H$, Cuvelier $C$, et al. Long-term evolution of gut inflammation in patients with spondyloarthropathy. Gastroenterology 1996; 110: 1696-1703.

3. Ciccia F, Accardo-Palumbo A, Alessandro R, et al. Interleukin-22 and interleukin-22-producing NKp44+ NK cells in the subclinical gut inflammation of patients with ankylosing spondylitis. Arthritis Rheum 2012; 64:1869-1878.

4. Ciccia F, Bombardieri M, Rizzo A, et al. Over-expression of paneth cell-derived antimicrobial peptides in the gut of patients with ankylosing spondylitis and subclinical intestinal inflammation. Rheumatology (Oxford) 2010; 49:20762083.

5. Ciccia F, Bombardieri M, Principato $A$, et al. Overexpression of interleukin-23, but not interleukin-17, as an immunologic signature of subclinical intestinal inflammation in ankylosing spondylitis. Arthritis Rheum 2009; 60:955-965.

6. Ciccia F, Lucchetti MM, Guggino G, et al. Gut dysbiosis in patients with HLA$\mathrm{B} 27+$ ankylosing spondylitis is associted with ileitis, down-regulation of tight junction proteins, increased serum levels of LPS and monocytes anergy. Ann Rheum Dis 2015; 74 (Suppl 2):148-149.

7. Mahida YR, Makh S, Hyde S, et al. Effect of Clostridium difficile toxin A on human intestinal epithelial cells: induction of interleukin 8 production and apoptosis after cell detachment. Gut 1996; 38:337-347.

8. Sanfilippo L, Baldwin TJ, Menozzi MG, et al. Heterogeneity in responses by primary adult human colonic epithelial cells to purified enterotoxin of Bacteroides fragilis. Gut 1998; 43:651-655.

9. Khor B, Gardet A, Xavier RJ. Genetics and pathogenesis of inflammatory bowel disease. Nature 2011; 474:307-317.

10. Dalal SR, Chang EB. The microbial basis of inflammatory bowel diseases. J Clin Invest 2014; 124:4190-4196.

11. Bowness P. HLA-B27. Annu Rev Immunol 2015; 33:29-48.

12. Lin $P$, Bach $M$, Asquith $M$, et al. HLA-B27 and human $\beta 2$-microglobulin affect the gut microbiota of transgenic rats. PLoS One 2014; 9:e105684. doi: 101371/journalpone0105684.

13. Vaile JH, Meddings JB, Yacyshyn BR, et al. Bowel permeability and CD45RO expression on circulating CD20+ B cells in patients with ankylosing spondylitis and their relatives. J Rheumatol 1999; 26:128-135.

14. Martínez-González $O$, Cantero-Hinojosa J, Paule-Sastre $P$, et al. Intestinal permeability in patients with ankylosing spondylitis and their healthy relatives. Br J Rheumatol 1994; 33:644-647.

15. Mielants $\mathrm{H}$, Veys $\mathrm{EM}$, De Vos $\mathrm{M}$, et al. Increased intestinal permeability in ankylosing spondylitis. Gut 1992; 33:1150.

16. Costello ME, Ciccia F, Willner $D$, et al. Intestinal dysbiosis in ankylosing

n. spondylitis. Arthritis Rheumatol 2015; 67: doi: 101002/art38967 [Epub ahead of print].

In this study, it is provided the first demonstration of a specific microbial signature

in the gut of AS patients

17. Stoll ML, Kumar R, Morrow $C D$, et al. Altered microbiota associated with abnormal humoral immune responses to commensal organisms in enthesitisrelated arthritis. Arthritis Res Ther 2014; 16:486.

18. Scher JU, Ubeda $C$, Artacho A, et al. Decreased bacterial diversity characterizes the altered gut microbiota in patients with psoriatic arthritis, resembling dysbiosis in inflammatory bowel disease. Arthritis Rheumatol 2015; 67:128-139.

19. Mantovani A, Biswas SK, Galdiero MR, et al. Macrophage plasticity and polarization in tissue repair and remodelling. J Pathol 2013; 229:176-185.

20. Smith PD, Smythies LE, Mosteller-Barnum M, et al. Intestinal macrophages lack CD14 and CD89 and consequently are down-regulated for LPS- and IgA-mediated activities. J Immunol 2001; 167:2651-2656.

21. Kamada N, Hisamatsu $T$, Okamoto $S$, et al. Unique CD14 intestinal macrophages contribute to the pathogenesis of Crohn disease via IL-23/IFNgamma axis. J Clin Invest 2008; 118:2269-2280.

22. Kamada $\mathrm{N}$, Hisamatsu $\mathrm{T}$, Honda $\mathrm{H}$, et al. Human CD14+ macrophages in intestinal lamina propria exhibit potent antigen-presenting ability. J Immunol 2009; 183:1724-1731.

23. Auffray C, Sieweke MH, Geissmann F. Blood monocytes: development, heterogeneity, and relationship with dendritic cells. Annu Rev Immunol 2009; 27:669-692.

24. Bystrom J, Evans I, Newson J, et al. Resolution-phase macrophages possess a unique inflammatory phenotype that is controlled by cAMP. Blood 2008; 112:4117-4127.

25. Vandooren B, Noordenbos $T$, Ambarus $C$, et al. Absence of a classically activated macrophage cytokine signature in peripheral spondylarthritis, including psoriatic arthritis. Arthritis Rheum 2009; 60:966-975.

26. Demetter $P$, De Vos $M$, Van Huysse JA, et al. Colon mucosa of patients both with spondyloarthritis and Crohn's disease is enriched with macrophages expressing the scavenger receptor CD163. Ann Rheum Dis 2005; 64:321324.

27. Appel H, Maier R, Bleil J, et al. In situ analysis of interleukin-23- and interleukin12 -positive cells in the spine of patients with ankylosing spondylitis. Arthritis Rheum 2013; 65:1522-1529.

28. Ciccia F, Alessandro R, Rizzo A, et al. Macrophage phenotype in the subclinical gut inflammation of patients with ankylosing spondylitis. Rheumatology (Oxford) 2014; 53:104-113.

29. Van Damme N, De Vos $M$, Baeten $D$, et al. Flow cytometric analysis of gut mucosal lymphocytes supports an impaired Th1 cytokine profile in spondyloarthropathy. Ann Rheum Dis 2001; 60:495-499. 
30. Sherlock JP, Taylor PC, Buckley CD, et al. Spondyloarthropathy: interleukin 23 and disease modification. Lancet 2015; 385:2017-2018.

31. Gaffen SL, Jain R, Garg AV, Cua DJ. The IL-23-IL-17 immune axis: from mechanisms to therapeutic testing. Nat Rev Immunol 2014; 14:585-600.

32. Hueber W, Sands BE, Lewitzky S, et al. Secukinumab, a human anti-IL-17A monoclonal antibody, for moderate to severe Crohn's disease: unexpected results of a randomised, double-blind placebo-controlled trial. Gut 2012; $61: 1693-1700$.

33. Bernink JH, Krabbendam L, Germar K, et al. Interleukin-12 and -23 control

I. plasticity of CD127(+) group 1 and group 3 innate lymphoid cells in the intestinal lamina propria. Immunity 2015; 43:146-160.

The study demonstrates that environmental cues determine the composition, function, and phenotype of CD127(+) ILC1 and ILC3 in the gut

34. Geremia A, Arancibia-Cárcamo CV, Fleming MP, et al. IL-23-responsive innate lymphoid cells are increased in inflammatory bowel disease. J Exp Med 2011; 208:1127-1133.

35. Peral de Castro C, Jones SA, Ní Cheallaigh C. Autophagy regulates IL-23 secretion and innate $\mathrm{T}$ cell responses through effects on IL-1 secretion. J Immunol 2012; 189:4144-4153.

36. Levine $B$. Eating oneself and uninvited guests: autophagy-related pathways in cellular defense. Cell 2005; 120:159-162.

37. Kruse KB, Brodsky JL, McCracken AA. Autophagy: an ER protein quality control process. Autophagy 2006; 2:135-137.

38. Ciccia F, Accardo-Palumbo A, Rizzo A, et al. Evidence that autophagy, but not

- the unfolded protein response, regulates the expression of IL-23 in the gut of patients with ankylosing spondylitis and subclinical gut inflammation. Ann Rheum Dis 2014; 73:1566-1574.

In this study there is the first evidence of HLA-B27 misfolding in AS and of autophagy involvement in IL-23 production in AS gut.

39. Neerinckx B, Carter B, Lories R. IL-23 expression and activation of autophagy in synovium and PBMCs of HLA-B27 positive patients with ankylosing spondylitis. Response to: 'Evidence that autophagy, but not the unfolded protein response, regulates the expression of IL-23 in the gut of patients with ankylosing spondylitis and subclinical gut inflammation' by Ciccia et al. Ann Rheum Dis 2014; 73:e68.

40. Turner MJ, Sowders DP, DeLay ML, et al. HLA-B27 misfolding in transgenic rats is associated with activation of the unfolded protein response. $\mathrm{J}$ Immunol $2005 ; 175: 2438-2448$.

41. Zeng L, Lindstrom MJ, Smith JA. Ankylosing spondylitis macrophage production of higher levels of interleukin-23 in response to lipopolysaccharide without induction of a significant unfolded protein response. Arthritis Rheum $2011 ; 63: 3807-3817$.

42. Haroon N, Guggino G, Zhang Z, et al. Autophagy and unfolded protein response: a fine balance that can influence the pathogenesis of ankylosing spondylitis and inflammatory bowel disease. Ann Rheum Dis 2015; 74 (Suppl 2):148.
43. Sherlock JP, Joyce-Shaikh B, Turner SP, et al. IL-23 induces spondyloarthropathy by acting on ROR- $\gamma \mathrm{t}+\mathrm{CD} 3+\mathrm{CD} 4-\mathrm{CD} 8$ - entheseal resident T cells. Nat Med 2012; 18:1069-1076.

44. Sonnenberg GF, Artis D. Innate lymphoid cells in the initiation, regulation and resolution of inflammation. Nat Med 2015; 21:698-708.

45. Hazenberg MD, Spits H. Human innate lymphoid cells. Blood 2014; 124:700-709.

46. Spits $H$, Artis D, Colonna $M$, et al. Innate lymphoid cells: a proposal for uniform nomenclature. Nat Rev Immunol 2013; 13:145-149.

47. Crellin NK, Trifari S, Kaplan CD. Human NKp44+IL-22+ cells and LTi-like cells constitute a stable RORC + lineage distinct from conventional natural killer cells. J Exp Med 2010; 207:281-290.

48. Mortha A, Chudnovskiy A, Hashimoto D, et al. Microbiota-dependent cross-

I. talk between macrophages and ILC3 promotes intestinal homeostasis. Science $2014 ; 343: 1249288$.

The study demonstrates that commensal microbes promote a crosstalk between innate myeloid and lymphoid cells that leads to gut immune homeostasis.

49. Baychelier F, Sennepin A, Ermonval M, et al. Identification of a cellular ligand for the natural cytotoxicity receptor NKp44. Blood 2013; 122:2935-2942.

50. Glatzer T, Killig M, Meisig J, et al. ROR $\gamma \mathrm{t}^{+}$innate lymphoid cells acquire a proinflammatory program upon engagement of the activating receptor NKp44. Immunity 2013; 38:1223-1235.

51. Ciccia F, Guggino G, Rizzo A, et al. Type 3 innate lymphoid cells producing IL-

- 17 and IL-22 are expanded in the gut, in the peripheral blood, synovial fluid and bone marrow of patients with ankylosing spondylitis. Ann Rheum Dis 2015; 74:1739-1747.

The study suggests the existence of an innate immune axis between the gut and the inflamed tissues of AS patients.

52. Eberl G. Inducible lymphoid tissues in the adult gut: recapitulation of a fetal developmental pathway? Nat Rev Immunol 2005; 5:413-420.

53. Knoop KA, Butler BR, Kumar N, et al. Distinct developmental requirements for isolated lymphoid follicle formation in the small and large intestine: RANKL is essential only in the small intestine. Am J Pathol 2011; 179:1861-1871.

54. Van Praet $L$, Jans $L$, Carron $P$, et al. Degree of bone marrow oedema in

- sacroiliac joints of patients with axial spondyloarthritis is linked to gut inflammation and male sex: results from the GIANT cohort. Ann Rheum Dis $2014 ; 73: 1186-1189$.

The study demonstrates the existence of a clinical link between subclinical gut inflammation and axial inflammation in AS.

55. Rehaume LM, Mondot $S$, Aguirre de Cárcer D, et al. ZAP-70 genotype disrupts the relationship between microbiota and host, leading to spondyloarthritis and ileitis in SKG mice. Arthritis Rheumatol 2014;66:2780-2792.

56. Benham $H$, Rehaume LM, Hasnain SZ, et al. Interleukin-23 mediates the intestinal response to microbial $\beta-1,3$-glucan and the development of spondyloarthritis pathology in SKG mice. Arthritis Rheumatol 2014; 66: 1755-1767. 